\title{
Mu-Negative Metamaterials Seen as Band- Limited Non-Foster Impedances in Inductive Power Transmission Systems
}

\author{
Jorge V. de Almeida ${ }^{1}$ (D), Gláucio L. Siqueira ${ }^{1}$ (D), Marbey M. Mosso ${ }^{1}$ (D), Carlos A. F. Sartori ${ }^{2,3}$ (D) \\ ${ }^{I}$ Dept. of Electrical Engineering, Center for Telecommunication Studies, Pontifical Catholic University of Rio \\ de Janeiro, Rio de Janeiro, RJ 22430-060, Brazil \\ virgilio@aluno.puc-rio.br, glaucio.siqueira@puc-rio.br,marbeyg@gmail.com \\ ${ }^{2}$ Dept. of Electrical Energy and Automation Engineering, Polytechnic School of University of São Paulo, São \\ Paulo, SP 05508-010, Brazil \\ ${ }^{3}$ Nuclear and Energy Research Institute, University of São Paulo, São Paulo, SP 03178-200, Brazil \\ sartori@pea.usp.br
}

\begin{abstract}
In the last decade, various works have demonstrated that a class of artificial material called metamaterials (MTM) can synthesize mu-negative (MNG) media capable of evanescent-wave focusing which largely enhances the magnetic coupling between coils, which is the basic mechanism of Inductive Power Transmission (IPT) systems. In the present work, MTM-enhanced coupling in IPT systems is examined through analytical and numerical results, which are validated by experimental data. Adopting a transmission-line (TL) based approach to describe the general MTM-enhanced IPT system, it is evidenced that MNG MTMs can be interpreted as a negative impedance from a circuital point of view.
\end{abstract}

Index Terms - Coupling enhancement; magnetic circuit; metamaterial-based lens; non-Foster impedance matching.

\section{INTRODUCTION}

The exploitation of evanescent waves in the near field for power transfer purpose has gained a lot of interest recently [1]. In order to prevent radiation, the drivers are high-Q electrically small antennas (ESA). Most of their energy remains stored in the surrounding near field and power transfer occurs primarily via induction. Nonetheless, such inductive power transmission is efficient only for distances smaller than the diameter of the antennas being also quite sensible to misalignment [2]. Electromagnetic MTM slabs presenting effective negative permeability can be used as near-field lenses to improve the overall efficiency of such inductive power transmission systems by enhancing the inductive coupling between the antennas. It has been called MTM-enhanced coupling [3]-[5].

Following the work previously presented in [6], this paper describes a transmission-line based approach of the interaction between a MNG MTM and a magnetic link. By introducing the concept of virtual magnetic transmission line (VMGTL) to describe the magnetic circuit and by calculating an equivalent virtual impedance to represent the MTM slab, the gain mechanism of those lenses can be regarded as a non-Foster impedance matching. Foster's reactance theorem states that any lossless passive one-port network has a reactance (or susceptance) that increases with frequency [7]. 
According to [8], non-Foster circuits can provide negative resistance, negative inductance and negative capacitance (meaning that their slope varies decreases with frequency). As it will be further shown, a passive MNG MTM behaves as a band-limited negative impedance described by a negative resistor in series with a negative inductor. The negative inductance implies on reduction of the amount of net energy stored in the magnetic circuit while the negative resistance implies on potential gain across the MTM.

This work is organized as follows: In Section II, a generalized theory of transmission lines is presented in order to include a magnetic coupled system. In Section III, equivalent virtual impedance for the proposed MTM slab is obtained. In Section IV and V, the analytic calculations and numerical results are presented and compared to demonstrate that a magnetic link can be described as VMGTL and that the gain associated with the MTM is due to a better impedance matching between the drivers. The results are supported by experimental evidence.

\section{GENERALIZED THEORY OF TRANSMISSION LINES}

\section{A. Physical and virtual transmission lines}

Most of the classical literature on TL is dedicated to electric transmission lines (ELTL), this is to say, the usage of two or multiple electric conductors (two-wire TL, coaxial cable, stripline and so on) to guide electromagnetic (EM) waves. Only recently, a broader concept of TLs has been proposed. As it has been demonstrated in [9], time-varying magnetic fluxes can guide EM fields like electric currents in ELTLs. In [10], it is proposed a generalization for TL theory that includes the flux-guided modes.

Fluxes and charges have the same dimension. It means that a time-varying flux will act as a sort of "virtual current" driving the fields:

$$
\begin{aligned}
j \omega \psi_{m} & =j \omega \iint \boldsymbol{B} \cdot d \boldsymbol{s}=\iint j \omega \boldsymbol{B} \cdot d \boldsymbol{s}=\iint \boldsymbol{J}_{m, d} \cdot d \boldsymbol{s}=I_{m, d} \quad[V] \\
j \omega \psi_{e} & =j \omega \iint \boldsymbol{D} \cdot d \boldsymbol{s}=\iint j \omega \boldsymbol{D} \cdot d \boldsymbol{s}=\iint \boldsymbol{J}_{e, d} \cdot d \boldsymbol{s}=I_{e, d} \quad[A]
\end{aligned}
$$

where $\psi_{m}$ and $\psi_{e}$ are, respectively, the magnetic and the electric fluxes, $\boldsymbol{B}$ and $\boldsymbol{D}$ the magnetic and electric flux densities, $\boldsymbol{J}_{m, d}$ and $\boldsymbol{J}_{e, d}$ the magnetic and electric displacement current densities, and $I_{m, d}$ and $I_{e, d}$ the total magnetic and the electric displacement currents.

Based on that fact, the phenomena of induction can be effectively described by TL equations by taking the displacement currents, the fluxes and the reluctance of the medium as virtual currents, virtual charges and virtual impedances, respectively. The TLs employing displacement currents to guide the EM fields are defined as virtual TLs while those employing conduction currents are defined as Physical TLs.

\section{B. Propagation equations for VMGTL}

For all purpose, a VMGTL consists on a magnetic circuit between two insulated drivers with no physical charge flowing between them and interacting only by means of inductive coupling. The 
transmitter is the magneto-motive force (MMF) source that generates the time-varying magnetic flux (or the displacement current) of the magnetic link while the receiver is the transducer that converts the magnetic flux to physical charges at the level of the load (see Fig. 1). In this work, the load is assumed to be a $50 \mathrm{Ohms}$ resistance.

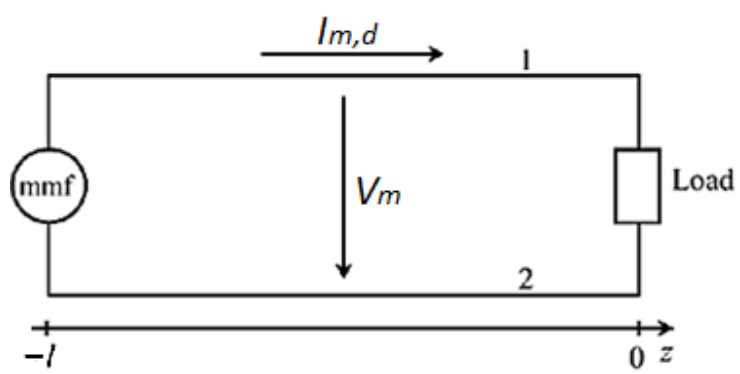

Fig. 1. Schematic of a VMGTL.

The total MMF of the virtual line is a real magnetic potential $V_{m}$ because fluxes going in opposite directions possess inverted magnetic potentials. Consequently, there must be a uniform magnetic field stored between them. By integration, the total $V_{m}$ of the VMGTL can be obtained:

$$
\operatorname{MMF}(\omega)=\psi_{m}(\omega) \mathcal{R}_{m}(\omega)=\int_{a}^{b} \boldsymbol{H}(\omega) \cdot d \boldsymbol{l}=V_{m}(\omega) \quad[A]
$$

Since the magnitude of the magnetic flux and the magnetic reluctance perceived by it are functions of the angular frequency $\omega, V_{m}$ is frequency-dependent. As it is shown in Fig. 2, the vector product of the magnetic field stored between the incoming and ongoing fluxes and the electric circulation generated by the time derivative of the flux is the very power-flow mechanism of the link [11], which implies it is frequency-dependent as well:

$$
P_{f}(\omega)=j \omega \mathcal{R}_{m} \psi_{m}^{2}(\omega)=V_{m} I_{m, d}(\omega) \quad[W]
$$

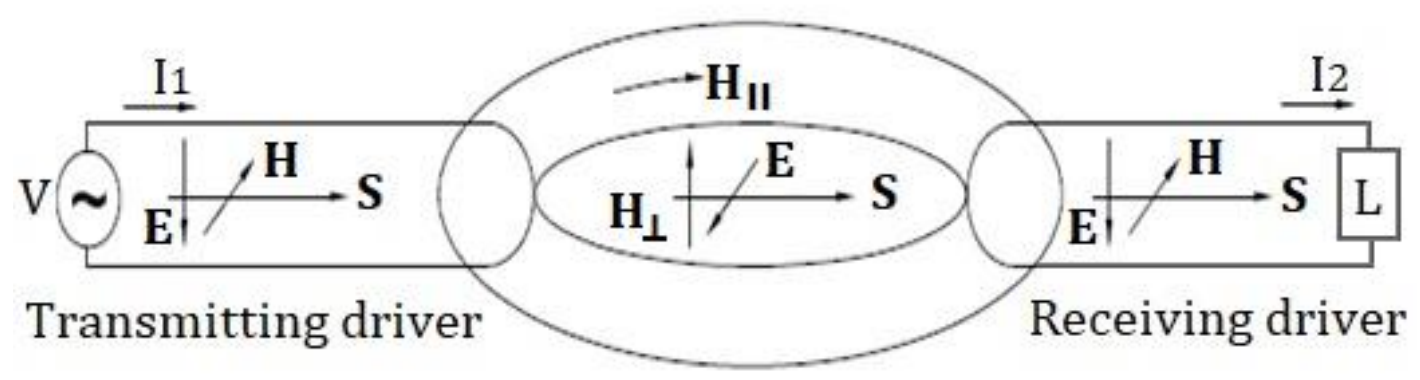

Fig. 2. The Poynting vector of the magnetic link depends on the transversal H-field component not the parallel one.

The magnetic potential $V_{m}$ and the magnetic virtual current $I_{m, d}$ of the VMGTL follow propagation equations analogous to the ones of conventional TLs:

$$
\begin{gathered}
\frac{d}{d z} V_{m}(z)=\left(\frac{\mathcal{R}_{m}^{\prime}}{j \omega}+G_{e}^{\prime}+j \omega C^{\prime}\right) I_{m, d}=Y_{m} I_{m, d}(z) \quad\left[\frac{A}{m}\right] \\
\frac{d}{d z} I_{m, d}(z)=\left(G_{m}^{\prime}+j \omega L^{\prime}\right) V_{m}=Z_{m} V_{m}(z) \quad\left[\frac{V}{m}\right]
\end{gathered}
$$

where $Z_{m}, Y_{m}, \mathcal{R}_{m}^{\prime}, G_{e}^{\prime}, G_{m}^{\prime}, L^{\prime}$ and $C^{\prime}$ denote the series electrical impedance, the shunt electrical admittance, the magnetic reluctance, the electrical conductance, the magnetic conductance, the inductance and the capacitance per unit length, respectively. 
Assuming the simplifying hypothesis of a magnetic flux confined in an invariant transversal section, the real and the imaginary parts of the magnetic reluctance $\mathcal{R}_{m}$ of the circuit can be approximated by [9]:

$$
\begin{gathered}
\operatorname{Re}\left\{\mathcal{R}_{m}(\omega)\right\}=2 \frac{\left(\bar{U}_{m}(\omega)-\bar{U}_{e}(\omega)\right)}{\psi_{m}^{2}(\omega)}\left[H^{-1}\right] \\
\operatorname{Im}\left\{\mathcal{R}_{m}(\omega)\right\}=\frac{P_{d, d}(\omega)}{\omega \psi_{m}^{2}(\omega)}\left[H^{-1}\right]
\end{gathered}
$$

where $\bar{U}_{m}$ and $\bar{U}_{m}$ are, respectively, the time-average magnetic and electric energies stored by the drivers and $P_{d, d}$ are the time-average power they dissipate.

From Eq. (5) and (6), it can be seen that the term $\frac{\mathcal{R}_{m}^{\prime}}{j \omega}$ is a virtual admittance due to the fact it neither stores nor dissipate any active power. Similarly to the radiation resistance of an antenna [12], it has no relationship with the thermal equilibrium of the circuit generating the flux. From this fact comes the result shown in Eq. (7) where the real part of $\mathcal{R}_{m}^{\prime}$ can be positive, negative or null depending on the balance of the energies stored in each field. In comparison, its imaginary part which accounts for the losses is shown to be always positive in Eq. (8).

The characteristic electrical impedance $Z_{0}$ of the VMGTL, its complex propagation parameter $\gamma$, its load reflection coefficient $\Gamma$ and its input impedance $Z_{\text {in }}$ can be determined by:

$$
\begin{gathered}
Z_{0}=\frac{I_{m, d}(z)}{V_{m}(z)}=\sqrt{\frac{Z_{m}}{Y_{m}}=\sqrt{\frac{G_{m}^{\prime}+j \omega L^{\prime}}{\mathcal{R}_{m}^{\prime}+G_{e}^{\prime}+j \omega C^{\prime}}}} \quad[\Omega] \\
\gamma=\sqrt{Z_{m} Y_{m}}=\sqrt{\left(\frac{G_{m}^{\prime}}{j \omega}+L^{\prime}\right)\left(-\frac{\mathcal{R}_{m}^{\prime}}{\omega^{2}}+\frac{G_{e}^{\prime}}{j \omega}+C^{\prime}\right)} \quad\left[\frac{\mathrm{rad}}{\mathrm{m}}\right] \\
Z_{\text {in }}=Z_{0} \frac{Z_{L}+Z_{L} \tanh (\gamma l)}{Z_{0}+Z_{L} \tanh (\gamma l)}
\end{gathered}
$$

The magnetic potential $V_{m}$ and the virtual current $I_{m, d}$ are associated with the electric potential $V_{e}$ and the electric current $I_{e}$ in the terminals through the following relationship:

$$
\begin{array}{ll}
V_{m}(-l)=V_{m}^{\text {in }}=I_{e}^{\text {in }} & {[A]} \\
I_{m, d}(-l)=I_{m, d}^{\text {in }}=V_{e}^{\text {in }} & {[\mathrm{V}]} \\
V_{m}(0)=V_{m}^{\text {out }}=I_{e}^{\text {out }} & {[\mathrm{A}]} \\
I_{m, d}(0)=I_{m, d}^{\text {out }}=V_{e}^{\text {out }} & {[\mathrm{V}]}
\end{array}
$$

Note that the magnetic and electric potentials and currents are dimensionally inverted. Since VMGTLs support transverse electromagnetic (TEM) modes as shown in [9], the propagation is a onedimensional Helmholtz problem [13]:

$$
\begin{array}{ll}
I_{m}(z)=I_{m}(0) e^{-\gamma z}\left(1+\Gamma e^{2 \gamma z}\right) & {[V]} \\
V_{m}(z)=\frac{I_{m}(0)}{Z_{0}} e^{-\gamma z}\left(1-\Gamma e^{2 \gamma z}\right) & {[A]}
\end{array}
$$

\section{Propagation equations for virtual magnetic transmission lines in free space}

If the flux is not channeled from the source to the load by a ferromagnetic material, the flux leakage of the VMGTL must be taken into consideration. Starting from the result presented in the previous 
section, the flux leakage is assumed to affect mainly the complex propagation constant $\gamma$. In order to compensate that, a numerically determined factor $\delta$ is introduced to adjust the phase variation and the attenuation of both the magnetic current and the magnetic potential of the VMGTL:

$$
\delta=\frac{0.23}{\kappa_{m}}
$$

where $\kappa_{m}$ is the magnetic coupling coefficient:

$$
\begin{aligned}
& \kappa_{m}=\frac{L_{m}}{\sqrt{L_{1} L_{2}}} \\
& I_{m}(z)=I_{m}(0) e^{-\delta \gamma z}\left(1+\Gamma_{m} e^{2 \delta \gamma z}\right) \quad[A] \\
& V_{m}(z)=\frac{I_{m}(0)}{Z_{0}} e^{-\delta \gamma z}\left(1-\Gamma_{m} e^{2 \delta \gamma z}\right) \quad[V]
\end{aligned}
$$

Hence, the overall gain introduced by the MTM can be defined directly from the power flow by calculating the difference between the transmission coefficient $S_{21}$ of circuit with and without the MTM slab:

$$
\begin{gathered}
P_{f}(z)=V_{m}(z) I_{m}^{*}(z) \quad[W] \\
S_{21, \text { analytical }}=\operatorname{sqrt}\left(\frac{P_{f}(0)}{P_{f}(-l)}\right) \\
\text { Gain }=\frac{S_{21, \text { with MTM }}}{S_{21, \text { without MTM }}}
\end{gathered}
$$

TABLE I. THE CONSTITUTIVE PARAMETERS OF THE MTM SLAB

\begin{tabular}{cc}
\hline Main parameters & Value \\
\hline$L_{\text {cell }}$ & $240 \mathrm{nH}$ \\
$f_{0}$ & $32.4 \mathrm{MHz}$ \\
$C=\frac{1}{\omega_{0}^{2} L}$ & $100 \mathrm{pF}$ \\
$Q_{\text {cell }}$ & 245 \\
Number of cells & 49 \\
Periodicity & $2.3 \mathrm{~cm}$ \\
\hline
\end{tabular}

\section{EQUIVALENT VIRTUAL IMPEDANCE FOR A MU-NEGATIVE METAMATERIAL}

In this section, the analytical representation of the virtual impedance of MNG MTM is compared with the one obtained through numerical simulation using the S-parameters to estimate the equivalent $\mu_{r}$ of the structure [14]. The numerical results were obtained on Keysight ADS 2016 using the Method of Moments (MoM). The proposed MTM to synthesize a MNG slab is the same model exploited in [15]. Its main characteristics are presented in Table I. Using the Lorentz model for EM materials, the relative permeability $\mu_{r}$ of the MTM can be estimated [16]:

$$
\mu_{r, M T M}=1+\frac{F \omega^{2}}{\omega^{2}-j \omega \xi-\omega_{0}^{2}} \quad\left[\frac{H}{m}\right]
$$

where $\xi$ is the damping ratio of the system and $F$ is the coupling coefficient between adjacent cells of the lattice. Note that $\mu_{r, M T M}$ will tend to 1 out of the resonance.

In Fig. 3, it can be seen that $\mu_{\mathrm{r}}$ becomes negative close to the resonance frequency in both the 
analytical model $\left(f_{0}=32.4 \mathrm{MHz}\right)$ and the numerical simulation $\left(f_{0}=32.4 \mathrm{MHz}\right)$. Although the overall behavior of the model and simulation are the same, the magnitudes, the Q-factor and the resonance frequency differs, which is attended due to the model's simplifications that underestimate the slab losses.

Using the effective value of $\mu_{\mathrm{r}}$, the equivalent inductance of the MTM can be determined:

$$
L_{M T M}=\mu_{0} a \mu_{r, M T M}(\omega)=\mu_{0} a\left(1+\frac{F \omega^{2}}{\omega^{2}-j \omega \xi-\omega_{0}^{2}}\right) \quad[H]
$$

Then, the MTM equivalent virtual impedance is given by:

$$
Z_{M T M}(\omega)=j X_{M T M}(\omega)=j \omega L_{M T M}(\omega) \quad[\Omega]
$$
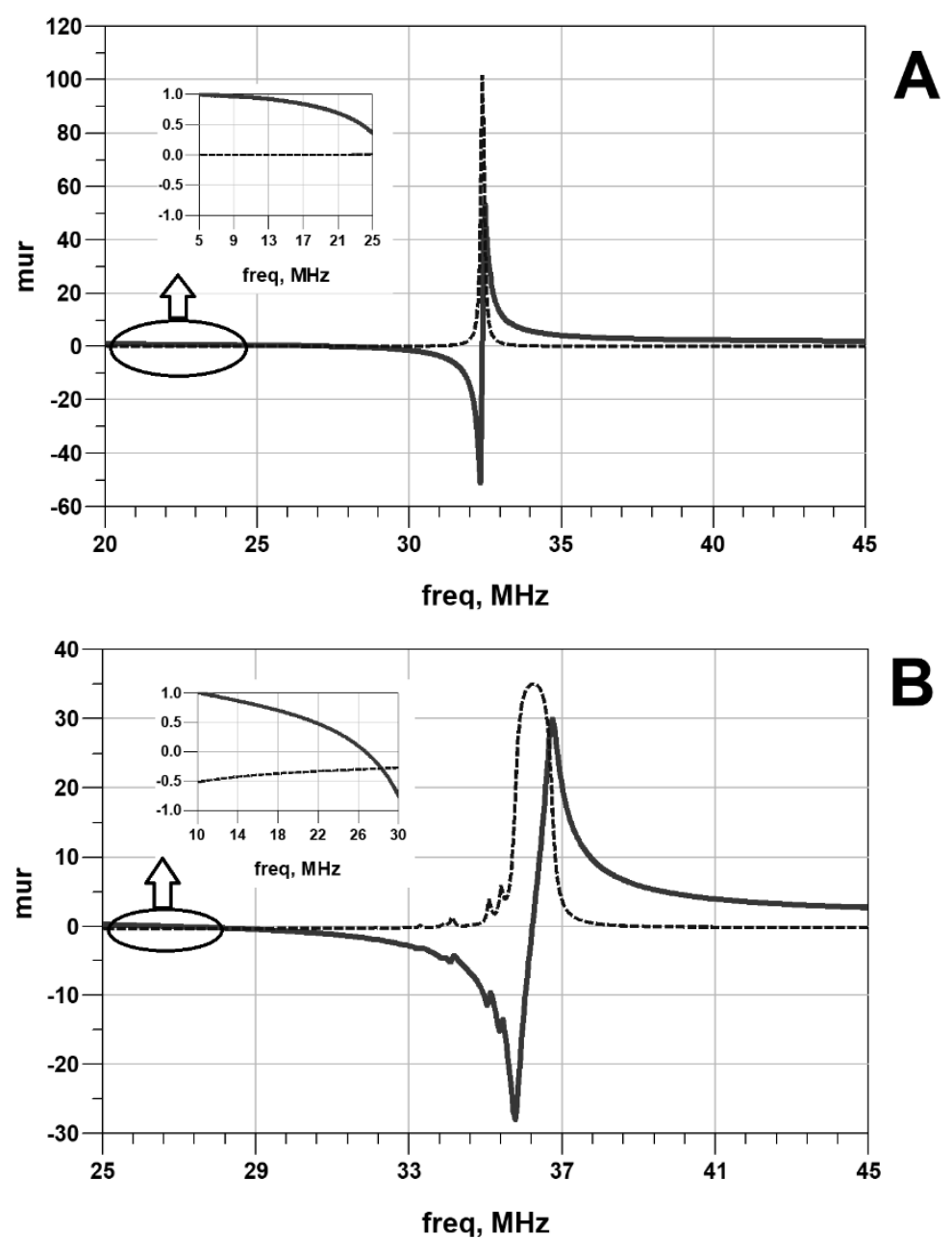

Fig. 3. Analytical (A) and numerically simulated (B) results for the real (solid) and imaginary part (dashed) of $\boldsymbol{\mu}_{\boldsymbol{r}}$.

Since the slope of $Z_{M T M}$ does not increase monotonically with the frequency, it can be said to possess a band-limited non-Foster behavior. As it is shown in Fig. 4, bellow the resonance the MTM is totally invisible to the virtual line $\left(Z_{M T M}=0\right)$. Around the resonance, it is perceived by the magnetic link as a negative resistance or an electric potential gain $\left(\operatorname{Re}\left\{Z_{M T M}\right\}<0\right)$ and a negative reactance or a capacitance $\left(\operatorname{Im}\left\{Z_{M T M}\right\}<0\right)$. Finally, above the resonance it is seen as a purely positive reactance or an inductance $\left(Z_{M T M} \in \mathrm{Im}^{+}\right)$. 

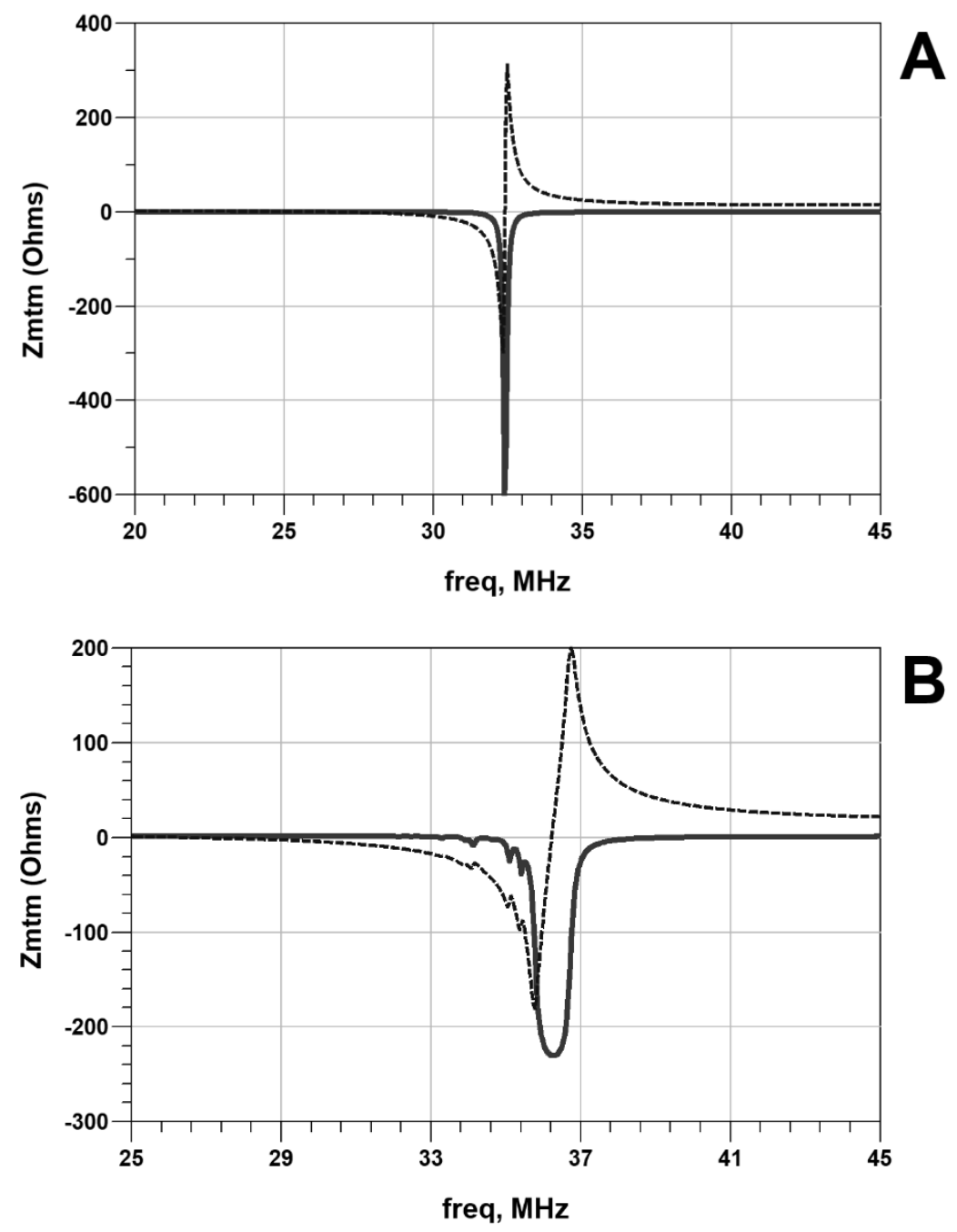

Fig. 4. Analytical (A) and numerically simulated (B) results for the real part (solid) and the imaginary part (dashed) of the MTM equivalent impedance $\boldsymbol{Z}_{\boldsymbol{M T M}}$.

When the MTM slab is seen as a negative resistance, it tends to enhance (to restore) the magnetic current of the VMGTL. As a negative reactance, it acts similarly to a negative inductance converting the stored magnetic energy into the complementary electric one, which reduces the total inductance of the circuit. Finally, as a pure positive reactance, it acts as conventional inductance and stores the surrounding magnetic field. From this comes the conclusion that the observed gain at the resonance is actually taken its surplus of energy from the attenuation of the higher frequencies modes that are being "trapped" by the MTM.

In the presence of the MTM slab, Eq. (6) can be rewritten as:

$$
\frac{d}{d z} I_{m, d}(z)=\left(G_{m}^{\prime}+j \omega L^{\prime}+Z_{M T M}\right) V_{m, v}(z) \quad\left[\frac{A}{m}\right]
$$

which is equal to a characteristic impedance $Z_{0}$ and complex propagation parameter $\gamma$ given by:

$$
Z_{0}=\sqrt{\frac{G_{m}^{\prime}+j \omega L^{\prime}+Z_{M T M}}{\frac{\mathcal{R}_{m}^{\prime}}{j \omega}+G_{e}^{\prime}+j \omega C^{\prime}}} \quad[\Omega]
$$




$$
\gamma=\sqrt{\left(\frac{G_{m}^{\prime}+Z_{M T M}}{j \omega}+L^{\prime}\right)\left(-\frac{\mathcal{R}_{m}^{\prime}}{\omega^{2}}+\frac{G_{e}^{\prime}}{j \omega}+C^{\prime}\right)} \quad\left[\frac{\mathrm{rad}}{m}\right]
$$

So, both $Z_{0}$ and $\gamma$ will not be altered below the resonance, it will tend to diminish around the resonance and it will tend to increase above the resonance.

\section{APPLICATION}

In order to validate the proposed model, the obtained results using the VMGTL formalism are compared with both numerical and experimental ones. The experimental data were obtained using a Vector Network Analyzer. The transmission and reflection coefficients were simulated on Keysight ADS 2016 using the MoM. The transversal magnetic field components were simulated on Keysight EMPro 2015 using the Finite-Difference Time-Domain (FDTD) method. The analytical results were a direct application of the virtual line model presented in section II.

The proposed free-space magnetic link consists of two loop antennas of radius $r=5 \mathrm{~cm}$ made of copper wire with diameter $p=1 \mathrm{~mm}$ assisted by the MTM described in section III. The loops operate far from self-resonance. It is assumed that the internal resistance of the source and the stray capacitance and the radiation resistance of the antennas are negligible. The secondary driver is connected to a load $R_{L}=50$ Ohms. The drivers are separated by a distance $D=15 \mathrm{~cm}$. The amplitude of the voltage source is $V_{e, c}^{i n}=1 \mathrm{~V}$. The loops are estimated to present a small resistance $R_{\text {source }}=0.5$ Ohms.

The self-inductance and the self-capacitance of the loops can be estimated by [17], [18]:

$$
\begin{gathered}
L_{0}=2 \mu_{0} r\left(\ln \left(\frac{16 r}{p}\right)-1.75\right)=620 n H \\
C_{0}=0.116 p+0.16 r+0.19 \sqrt{\frac{r}{p}}=0.28 p F
\end{gathered}
$$

The distributed inductance and capacitance of the line can be approximated by:

$$
\begin{aligned}
& L^{\prime}=\frac{L_{0}}{D} \quad\left[\frac{H}{m}\right] \\
& C^{\prime}=\frac{C_{0}}{D} \quad\left[\frac{F}{m}\right]
\end{aligned}
$$

For loops magnetically coupled through the air:

$$
\begin{gathered}
\mu=\mu_{0} \rightarrow G_{m}^{\prime}=0 \\
\varepsilon=\varepsilon_{0} \rightarrow G_{e}^{\prime}=0
\end{gathered}
$$

Once the air has no intrinsic material loss, the reluctance of the VMGTL is purely real:

$$
\begin{gathered}
\mu^{\prime \prime}=\varepsilon^{\prime \prime}=0 \rightarrow \operatorname{Im}\left\{\mathcal{R}_{m}\right\}=0 \\
\mathcal{R}_{m}=\operatorname{Re}\left\{\mathcal{R}_{m}\right\}=2 \frac{\left(\bar{U}_{m}-\bar{U}_{e}\right)}{\psi_{m}^{2}}=2\left(\frac{1}{L_{0}}-\omega^{2} C_{0}\right) \quad\left[H^{-1}\right] \\
\mathcal{R}_{m}^{\prime}=\frac{\mathcal{R}_{m}}{D}\left[\frac{H^{-1}}{m}\right]
\end{gathered}
$$

The coupling coefficient between the antennas can be estimated by [19]:

$$
\kappa_{m} \approx \frac{\mu_{0} \pi r^{4}}{L_{0} \sqrt{4 r^{2}+D^{2}} D^{2}}=0.01
$$




\section{RESULTS AND DISCUSSION}

As it is shown in Fig. 5 and Fig. 6, the magnetic potential difference between the fluxes inside the link gives rise to a transversal component of the magnetic field $H_{\perp}$. When the MTM is activated line in Fig. 6, the distribution of $H_{\perp}$ and consequently the distribution of $V_{m}$ becomes much more uniform along the line.

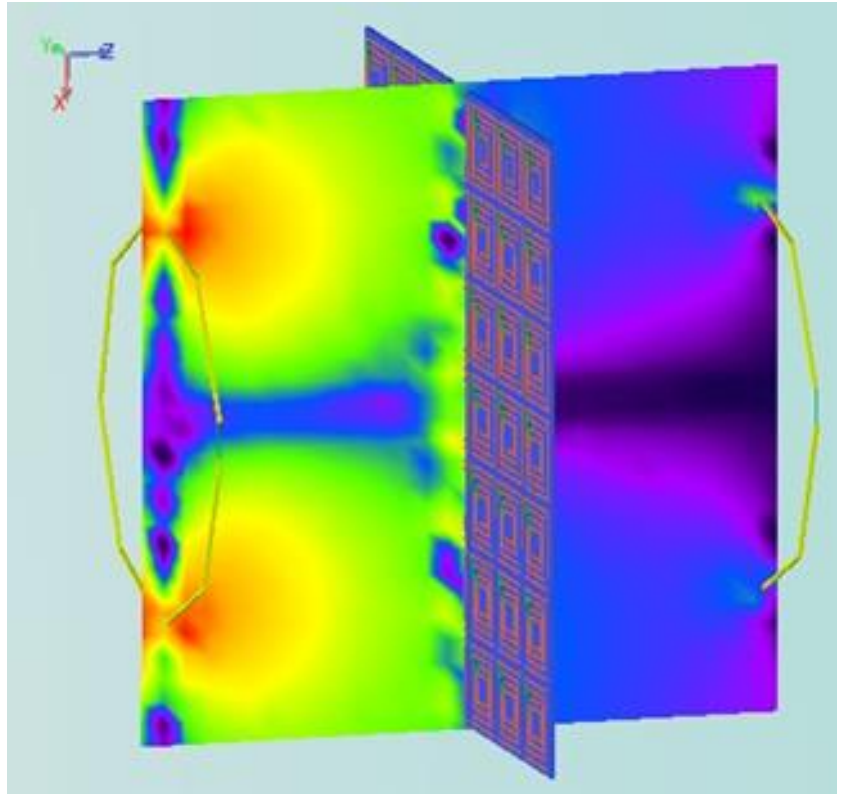

Fig. 5. Module of $\boldsymbol{H}_{\perp}$ out of the resonance at the plane XZ.

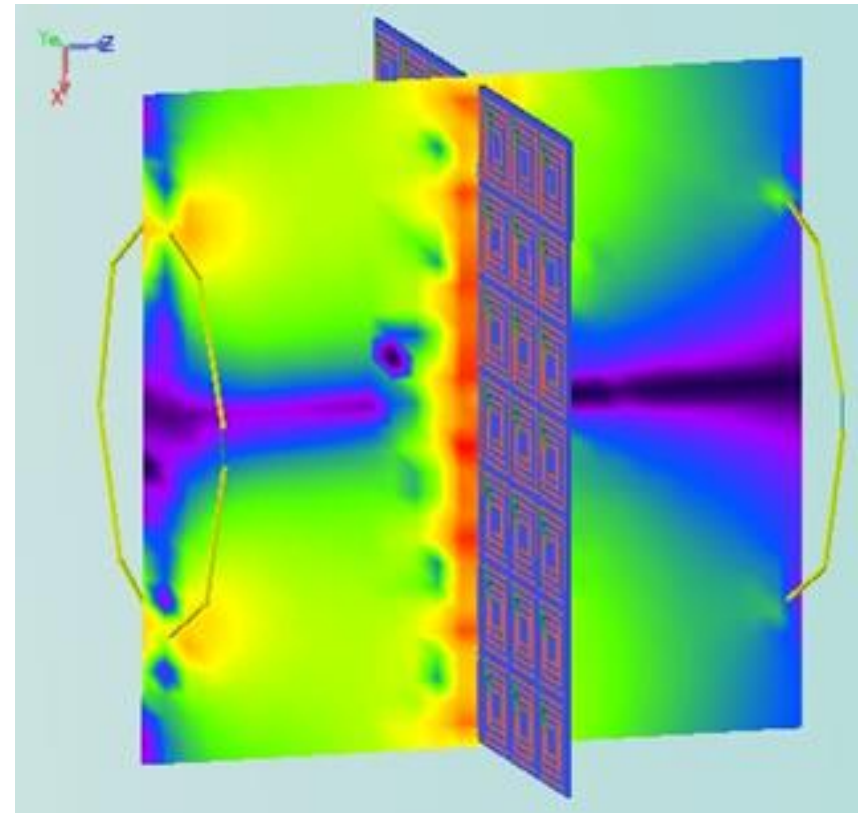

Fig. 6. Module of $\boldsymbol{H}_{\perp}$ around the resonance at the plane XZ. The magnetic potential between the coils becomes much more uniformly distributed in the presence of the MTM slab.

As shown in Fig. 7 and Fig. 8, the homogenization of the potential distribution inside the magnetic link is equivalent to an improved impedance matching in the TL theory point of view. 


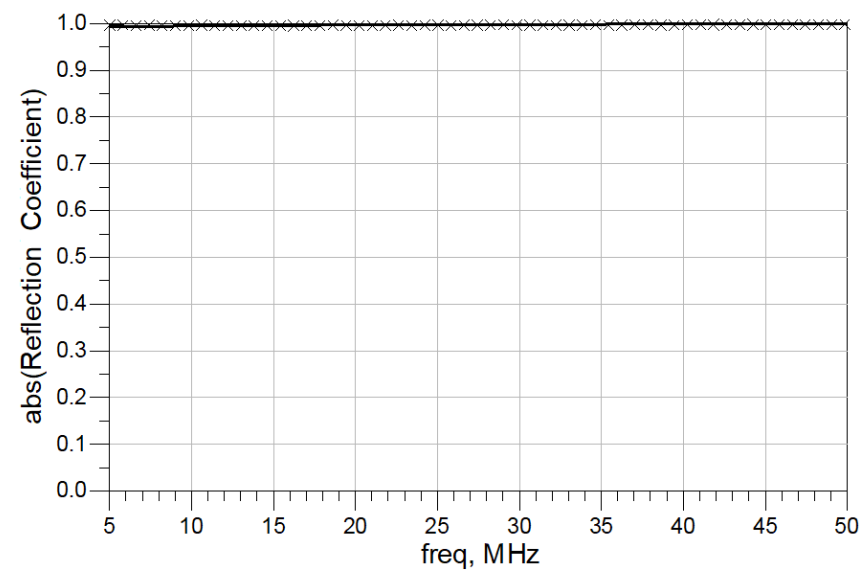

Fig. 7. Analytical (solid) and numerical (cross) estimations of the magnitude of $\boldsymbol{\Gamma}$ without the MTM slab.

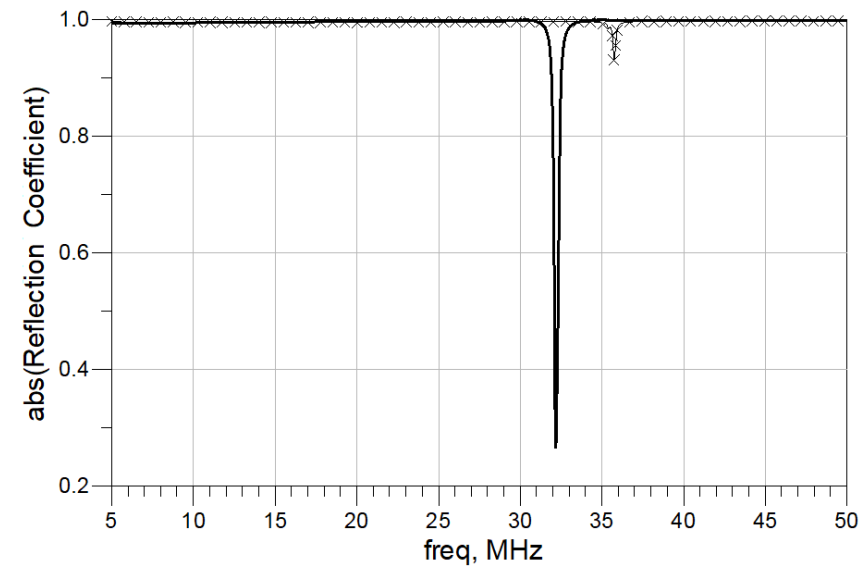

Fig. 8. Analytical (solid) and numerical (cross) estimations of the magnitude of $\boldsymbol{\Gamma}$ with the MTM slab.

Another important aspect of a better distribution of $V_{m}$ concerns the accuracy of the model. As it can be seen in Fig. 9 and Fig. 10, the model can describe the overall behavior of the magnetic link with and without the MTM. Nonetheless, it is much more accurate when the MTM is inside the magnetic link. The enhancement of the accuracy of the model is a consequence of the magnetic flux focusing induced by the MTM. It makes more realistic the hypothesis of magnetic flux confined to a path with constant transversal section.

Concerning the gain introduced by the MTM, the analytical prediction is quite optimistic in comparison with numerical simulation (see Table II). It is a consequence of the simplifications adopted by the model. However, even if the magnitude of the gain is overestimated by the model, the overall behavior of the system predicted by it shows a good agreement with the experimental one (see Fig. 11).

At last, it must be stressed out that the relative position of the MTM to the transmitting driver has not modified the transmission gain. This result reinforces that the interaction between the magnetic link and the MTM slab has a TL-like behavior. 
Journal of Microwaves, Optoelectronics and Electromagnetic Applications, Vol. 18, No. 4, December 2019502 DOI: http://dx.doi.org/10.1590/2179-10742019v18i41625

TABLE II. COMPARISON BETWEEN THE ANALYTICAL, SIMULATED AND EXPERIMENTAL RESULTS FOR THE GAIN

\begin{tabular}{cc}
\hline & Gain $(\mathbf{d B})$ \\
\hline Analytical & 29.2 \\
Numerical & 15 \\
Experimental & 9 \\
\hline
\end{tabular}

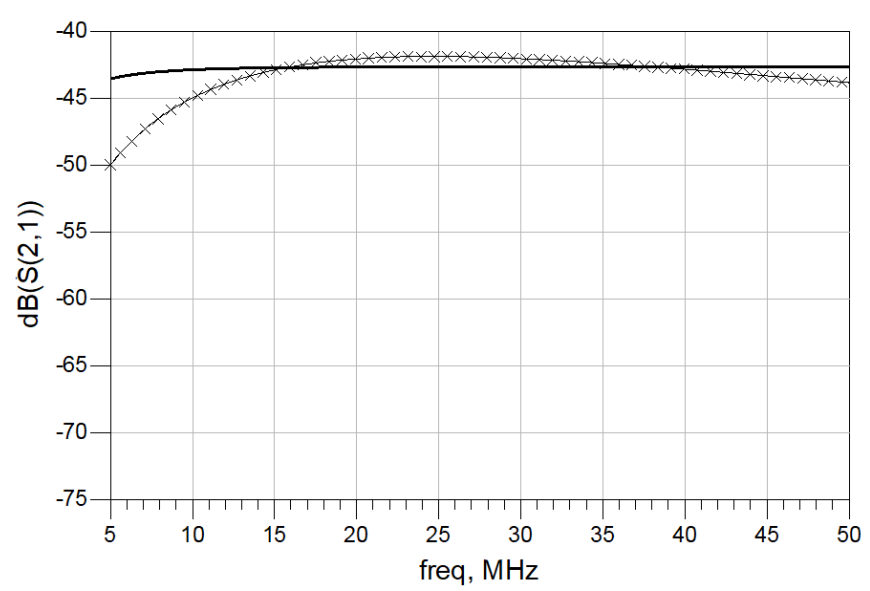

Fig. 9. Analytical (solid) and numerical (cross) estimations of the magnitude of $\boldsymbol{S}_{\mathbf{2 1}}$ without the MTM slab.

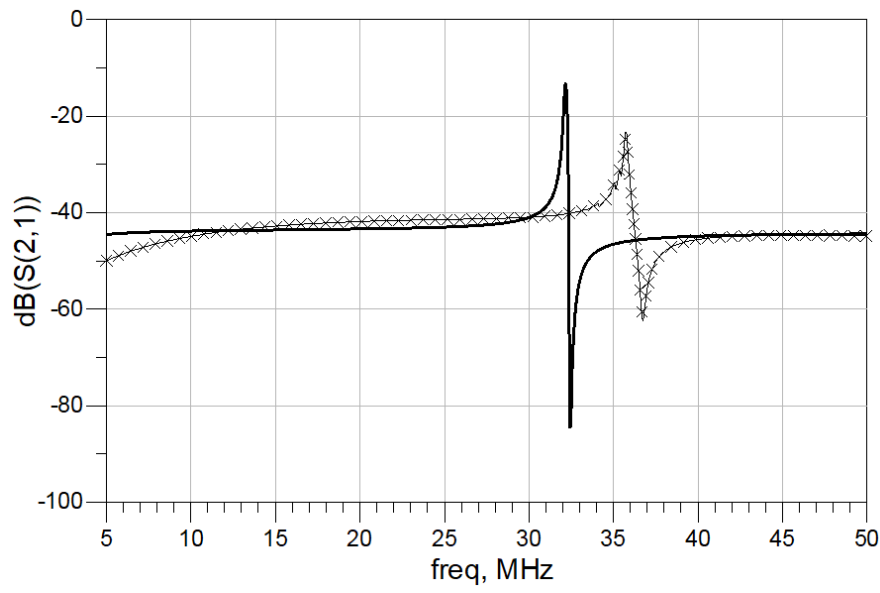

Fig. 10. Analytical (solid) and numerical (cross) estimations of the magnitude of $\boldsymbol{S}_{\mathbf{2 1}}$ with the MTM slab.

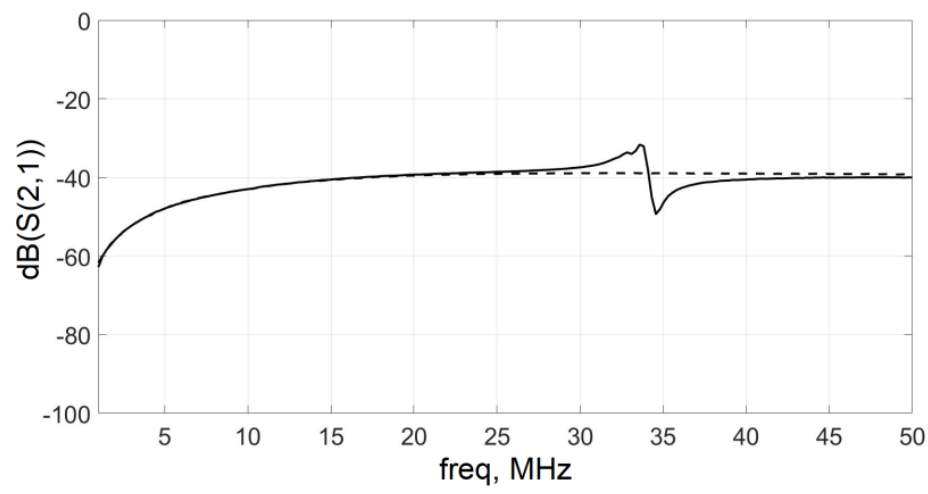

Fig. 11. Experimental result for the $\boldsymbol{S}_{\mathbf{2 1}}$ with (solid) and without (dashed) the MTM slab. 
Journal of Microwaves, Optoelectronics and Electromagnetic Applications, Vol. 18, No. 4, December 2019503 DOI: http://dx.doi.org/10.1590/2179-10742019v18i41625

\section{CONCLUSION}

In this work, it has been proposed the usage of a generalized TL theory to include the phenomenon of induction following the previous efforts presented by [9]. The concept of virtual currents and charges were introduced to well distinguish between flux-guided EM modes from charge-guided EM modes. It had been demonstrated that free-space magnetic coupling can also be described as virtual TL and that a MTM can be interpreted as a virtual impedance added to this circuit.

It has been shown that the amplification effect is not contradictory with the law of conservation of energy since it results from the exchange of energy between the reactive modes in the near field of the loops. The MTM attenuates the higher-frequency modes and transfers the surplus power to the resonant one. It means that the MTM acts as a band-limited gain medium around its resonance.

Since the transmission improvement is a consequence of the reduction of the virtual-line reactive power, any element interacting with the magnetic link causing the very same effect in terms of the impedance seen by the VMGTL (i.e., a resonant coil) would produce a similar transmission gain as the one presented by this particular MTM. This fact seems to confirm the results presented in [20].

Finally, the analytical model was verified through numerical and experimental results of a given magnetic link. The model confirms that the MTM gain comes from the improvement of the impedance matching between the transmitter and the receiver due to the non-Foster behavior of its equivalent virtual impedance close to its operating frequency. Although the model successfully predicts the behavior of the system and the underline mechanism of enhancement of power transfer due to the MTM slab, it is not accurate concerning the magnitude of these results. This lack of accuracy is mainly due to the non-deterministic nature of the magnetic potential of non-confined VMGTL. In the future, the introduction of a probabilistic description of the magnetic link would solve the accuracy related problems.

\section{REFERENCES}

[1] I. Mayordomo, T. Dräger, P. Spies, J. Bernhard and A. Pflaum, "An Overview of Technical Challenges and Advances of Inductive Wireless Power Transmission," Proceedings of the IEEE, vol. 101, no. 6, pp. 1302-1311, 2013.

[2] A. A. Eteng, S. K. A. Rahim and C. Y. Leow, "Wireless Nonradiative Energy Transfer: Antenna performance enhancement techniques," IEEE Antennas and Propagation Magazine , vol. 57, no. 3, pp. 16-22, 2015.

[3] Y. Urzhumov and D. R. Smith, "Metamaterial-enhanced coupling between magnetic dipoles for efficient wireless power transfer," Phys. Rev. B, vol. 83, no. 20, pp. 205114 1-10, 2011.

[4] D. Huang, Y. Urzhumov, D. R. Smith and K. H. Teo, "Magnetic superlens-enhanced inductive coupling for wireless power transfer," Journal of Applied Physics, vol. 111, no. 6, pp. 064902 1-8, 2012.

[5] J. V. de Almeida and R. S. Feitoza, "Metamaterial-Enhanced Magnetic Coupling: An Inductive Wireless Power Transmission System Assisted by Metamaterial-Based Mu-Negative Lenses," IEEE Microwave Magazine, vol. 19, no. 4, pp. 95 - 100, 2018.

[6] J. V. de Almeida, G. L. Siqueira, M. M. Mosso and C. A. F. Sartori, "Mu-negative metamaterials seen as band-limited non-Foster impedances for magnetic coupled systems," in Meta'17 Conference Proceedings, Incheon, 2017.

[7] R. Foster, "A Reactance Theorem," Bell System Technical Journal, vol. 3, no. 2, pp. 259-267, 1924.

[8] R. C. Hansen and R. E. Collin, Small Antenna Handbook, New Jersey: John Wiley and Sons, 2011, pp. 39-59.

[9] J. A. B. Faria and M. P. Pires, "Theory of Magnetic Transmission Lines," IEEE Transactions on Microwave Theory and Techniques, vol. 60, no. 10, pp. 2941-2949, 2012.

[10] J. V. de Almeida, "Virtual magnetic transmission lines," M.Sc. Eng. thesis, Pontifical Catholic Univ. Rio de Janeiro, 
Journal of Microwaves, Optoelectronics and Electromagnetic Applications, Vol. 18, No. 4, December 2019504 DOI: http://dx.doi.org/10.1590/2179-10742019v18i41625

Rio de Janeiro, Brazil, 2017.

[11] J. A. B. Faria, "Poynting Vector Flow Analysis for Contactless Energy Transfer in Magnetic Systems," IEEE Transactions on Power Electronics, vol. 27, no. 10, pp. 4292-4300, 2012.

[12] S. Banerjee, K. Das and B. Rana, Communication Engineering II, New Delhi: Vikas Publishing House, 2015, p. 91.

[13] R. F. Harrington, Time-Harmonic Electromagnetic Fields, Piscataway, NJ: Wiley-IEEE Press, 2001, pp. 61-66.

[14] E. Ekmekçi and G. Turhan-Sayan, "Investigation of effective permittivity and permeability for a novel v-shaped metamaterial using simulated s-parameters," in 5th International Conference on Electrical and Electronics Engineering, 2007.

[15] S. I. Nishimura, J. V. de Almeida, C. Vollaire, C. A. F. Sartori, A. Breard, F. Morel and L. Krähenbühl, "Enhancing the inductive coupling and efficiency of wireless power transmission system by using metamaterials," in $16^{\circ} S B M O$ Simpósio Brasileiro de Micro-ondas e Optoeletrônica e $11^{\circ}$ CBMag, Curitiba, 2014.

[16] R. W. Ziolkowski and N. Engheta, Electromagnetic Metamaterials: Physics and Engineering Explorations, Piscataway: Wiley-IEEE Press, 2006, pp. 88-91.

[17] "Antenna Theory," [Online]. Available: http://www.antenna-theory.com/antennas/smallLoop.php. [Accessed 2901 2016].

[18] R. G. Medhurst, "H. F. Resistance and Self-Capacitance of Single-Layer Solenoids," Wireless Engineer, pp. 35-43, 1947.

[19] R. M. Duarte and G. K. Felic, "Analysis of the Coupling Coefficient in Inductive Energy Transfer Systems," Active and Passive Electronic Components, vol. 2014, Article ID 951624, 6 pages, 2014.

[20] M. J. Chabalko, J. Besnoff and D. S. Ricketts, "Magnetic Field Enhancement in Wireless Power With Metamaterials and Magnetic Resonant Couplers," IEEE Antennas and Wireless Propagation Letters, vol. 15, pp. 452-455, 2015. 